\title{
ASYMPTOTIC PROPERTIES OF THE PLANE SHEAR THICKENING FLUIDS WITH BOUNDED ENERGY INTEGRAL
}

\author{
SHUAI LI, TAO WANG, AND WENDONG WANG
}

\begin{abstract}
In this note we investigate the asymptotic behavior of plane shear thickening fluids around a bounded obstacle. Different from the Navier-Stokes case considered by Gilbarg-Weinberger in [18], where the good structure of the vorticity can be exploited and weighted energy estimates can be applied, we have to overcome the nonlinear term of high order. The decay estimates of the velocity was obtained by combining Point-wise Behavior Theorem in [16] and Brezis-Gallouet inequality in [7] together, which is independent of interest.
\end{abstract}

\section{INTRODUCTION}

As Ladyzhenskaya suggests in her monograph in [20], it is interesting to investigate "new equations for the description of the motion of viscous incompressible fluids", which roughly speaking means to consider viscosity coefficients, which depend on the modulus of the symmetric gradient,

$$
\varepsilon(u)=\frac{1}{2}\left(D u+(D u)^{T}\right)=\frac{1}{2}\left(\partial_{i} u_{k}+\partial_{k} u_{i}\right)_{1 \leq i, k \leq 2}
$$

of the velocity field $u$, for example, in a monotonically increasing way (shear thickening case). In this note we will consider this problem in a very special situation restricting ourselves to stationary flows through an exterior domain $\Omega \subset \mathbb{R}^{2}$ with smooth boundary $\partial \Omega$. More precisely, consider the solution $u: \Omega \rightarrow \mathbb{R}^{2}, \pi: \Omega \rightarrow \mathbb{R}$ of the following system

$$
\left\{\begin{array}{l}
-\operatorname{div}[T(\varepsilon(u))]+u_{k} \partial_{k} u+D \pi=0, \text { in } \Omega, \\
\operatorname{div} u=0, \text { in } \Omega,
\end{array}\right.
$$

where the $\Omega=\mathbb{R}^{2} \backslash \overline{B_{R_{0}}(0)}$. More details on viscous incompressible flow, we refer to $[3,19,10,12,14,15,20]$ and the references therein.

The system (1.1) describes the stationary flow of an incompressible generalized Newtonian fluid, where $u$ is the velocity field, $\pi$ is the pressure function, $u_{k} \partial_{k} u$ is the convective term, and $T$ represents the stress deviator tensor. And we use $\varepsilon(u)$ to stand for the symmetric part of the differential matrix $D u$ of $u$. We assume that

Date: Dec 10, 2019.

2010 Mathematics Subject Classification. 35Q30, 76D03.

Key words and phrases. asymptotic behavior; shear thickening fluids; generalized Navier-Stokes equations; 
the stress tensor $T$ is the gradient of a potential $H: S^{2 \times 2} \rightarrow \mathbb{R}$ defined on the space $S^{2 \times 2}$ of all symmetric $2 \times 2$ matrices of the following form

$$
H(\varepsilon)=h(|\varepsilon|),
$$

where $h$ is a nonnegative function of class $C^{3}$. Thus

$$
T(\varepsilon)=D H(\varepsilon)=\mu(|\varepsilon|) \varepsilon, \mu(t)=\frac{h^{\prime}(t)}{t} .
$$

Note that the Navier-Stokes equations for incompressible Newtonian fluids follow from the system (1.1) if $\mu$ is a constant. If $\mu$ is not a constant, it means that the viscosity coefficient depends on $\varepsilon$, and system (1.1) describes the motion of continuous media of generalized Newtonian fluids.

As in [11], assume that the potential $h$ satisfies the follow conditions:

$$
\begin{gathered}
h \text { is strictly increasing and convex } \\
\text { together with } h^{\prime \prime}(0)>0 \text { and } \lim _{t \rightarrow 0} \frac{h(t)}{t}=0 ;
\end{gathered}
$$

there exist a constant $a \geq 1$ such that $h(2 t) \leq a h(t)$ for all $t \geq 0$;

$$
\frac{h^{\prime}(t)}{t} \leq h^{\prime \prime}(t) \text { for any } t \geq 0 \text {. }
$$

Let us sketch some progress on the system (1.1). First, the existence of strong solutions is proved in a bounded domain by Fuchs in [10]. The existence of Dirichlet energy solutions satisfying the boundary condition at infinity in an exterior is very difficult, even if for the Navier-Stokes equations, which is related to Leray's question; for example see Leray [21], Amick [1], Russo [24], Pileckas-Russo [23] and the references therein. Different from the Navier-Stokes case, the regularity is also unknown for general form $h(t)$ as in (1.2). Bildhauer-Fuchs-Zhong [6] proved the weak solution is $C^{1, \alpha}$ by assuming $h(t)=t^{2}(1+t)^{m}$, see also recent improved result by Jin-Kang in [19]. The Liouville property of (1.1) was started by Fuchs in [11], and later studied by Zhang in [25] and [26], where they obtained the trivial property of the solution with the help of $u \in L^{\infty}$ or $\int_{\Omega} h(|\nabla u|)<\infty$. The degenerate case $h(t)=t^{p}$ was also considered by Bildhauer-Fuchs-Zhang in [5] by assuming that $\int_{\Omega}|\nabla u|^{p}<\infty$. More developments, we refer to [5, 10] and the references therein.

In this note, motivated by the work of Gilbarg-Weinberger [18], we investigate the asymptotic properties of the solutions of (1.1). In [18], they showed that pressure function $\pi$ has a limit at infinity, $u(z)=o\left(\ln ^{\frac{1}{2}} r\right)$, and $|D u| \leq o\left(r^{-\frac{3}{4}}(\ln r)^{\frac{9}{8}}\right)$ provided that the Dirichlet energy is bounded in an exterior domain, i.e., $\int_{\Omega}|D u|^{2} d x<\infty$. Their proof relies on the fact that the vorticity of the 2D Navier-Stokes equations satisfies a nice elliptic equation, to which the maximum principle applies. Here we consider the case of shear thickening fluids, for $h$ satisfying the (A1)-(A3); however, it's difficult to exploit the good structure of the vorticity and apply weighted energy estimates, since the main part in (1.1) is nonlinear. Inspired by Point-wise Behavior Theorem in [16] and Brezis-Gallouet inequality (for example, see [7] or [8], we obtain 
the higher energy estimates, which imply the decay estimates by combining point-wise behavior theorem and Brezis-Gallouet inequality together.

As show in [5], the following properties of functions $h$ follows from (A1)-(A3).

(i) $\mu(t)=\frac{h^{\prime}(t)}{t}$ is an increasing function.

(ii) We have $h(0)=h^{\prime}(0)=0$ and

$$
h(t) \geq \frac{1}{2} h^{\prime \prime}(0) t^{2}, \quad h^{\prime \prime}(0)>0
$$

(iii) There exists a constant $a>0$ such that the function $h$ satisfies the balancing condition,

$$
\frac{1}{a} t h^{\prime}(t) \leq h(t) \leq t h^{\prime}(t), t \geq 0
$$

(iv) From the assumptions on $h$, we know the system satisfies the following elliptic condition, $\forall \varepsilon, \sigma \in S^{2}$,

$$
\frac{h^{\prime}(|\varepsilon|)}{|\varepsilon|}|\sigma|^{2} \leq D^{2} H(\varepsilon)(\sigma, \sigma) \leq h^{\prime \prime}(|\varepsilon|)|\sigma|^{2},
$$

from which, together with (1.3) and (1.4), it follows that

$$
D^{2} H(\varepsilon)(\sigma, \sigma) \geq \frac{1}{2} h^{\prime \prime}(0)|\sigma|^{2} .
$$

Let $T_{r}=B_{r}(0) \backslash \overline{B_{R_{0}}(0)}$ for any $r>R_{0}>0$. Our first result is to estimate the $L^{2}$ norm of $D^{2} u$.

Proposition 1.1. For $\Omega=\mathbb{R}^{2} \backslash \overline{B_{R_{0}}(0)}$, let $u \in C^{2}\left(\Omega, \mathbb{R}^{2}\right)$ be a solutions of (1.1). Then there hold $\int_{\Omega}\left|D^{2} u\right|^{2} d x<\infty$,

$$
\int_{T_{\frac{3}{2} r} \backslash T_{\frac{9}{8} r}}\left|D^{2} u\right|^{2} d x \leq C \frac{\sqrt{\log r}}{r},
$$

and

$$
|u(x)|=o(\sqrt{\ln (|x|)}),
$$

provided that $r=|x|$ is large enough and $\int_{\Omega} h(|D u|) d x<\infty$.

Remark 1.1. The $L^{2}$ norm of $D^{2} u$ was obtained in [25] in the whole space $\mathbb{R}^{2}$,

$$
\int_{B_{R}}\left|D^{2} u\right|^{2} d x \leq C+C \frac{1}{R^{3}} \int_{B_{2 R}}|u|^{2} d x
$$

Here we refine the estimate, especially for the exterior domain. One observation is to apply the Wirtinger's inequality in $L^{3}$ norm and another technique is to use Poincaré-Sobolev inequality in a circular region. The estimate (1.8) is the same as the Navier-Stokes case in [18]. 
In order to obtain the decay estimate of $D u$, we need to estimate the norm of $D^{3} u$. Generally, it's more difficult. Motivated by the anisotropic variational problems in [2, 4], we give the following assumption.

(v) Assume that

$$
1+C_{1} t^{\gamma_{1}} \leq h^{\prime \prime}(t) \leq 1+C_{2} t^{\gamma_{2}}, t \geq 0
$$

where $\gamma_{2} \geq \gamma_{1} \geq 1$ and $C_{2}>C_{1}>0$. From this, it follows that

$$
\begin{aligned}
& t^{2}+C_{1}^{\prime} t^{2+\gamma_{1}} \leq h(t) \leq t^{2}+C_{2}^{\prime} t^{2+\gamma_{2}}, \\
& t+C_{1}^{\prime \prime} t^{1+\gamma_{1}} \leq h^{\prime}(t) \leq t+C_{2}^{\prime \prime} t^{1+\gamma_{2}}, \quad t \geq 0,
\end{aligned}
$$

where $C_{1}^{\prime}, C_{2}^{\prime}, C_{1}^{\prime \prime}, C_{2}^{\prime \prime}$ depend on $C_{1}, C_{2}, \gamma_{1}$ and $\gamma_{2}$.

(vi) Assume that

$$
h^{\prime \prime \prime}(t) \leq C_{3}+C_{3}^{\prime} t^{\gamma_{0}}, \quad \gamma_{0} \geq 0, \quad t \geq 0 .
$$

where $C_{3}, C_{3}^{\prime} \geq 0$.

Proposition 1.2. Let $\Omega=\mathbb{R}^{2} \backslash \overline{B_{R_{0}}(0)}, u \in C^{3}\left(\Omega, \mathbb{R}^{2}\right)$ is a solutions of (1.1), if $|\varepsilon(u)| \leq C$, then there exists a constant $r_{0}>0$ such that

$$
\int_{T_{\frac{3}{2} r} \backslash T_{\frac{9}{8} r}}\left|D^{3} u\right|^{2} d x \leq C
$$

for any $r>r_{0}$, provided that $\int_{\Omega} h(|D u|) d x<\infty$.

Remark 1.2. The conditions (v) and (vi) are used to estimate the term $J_{3}$ in Sec. 4:

$$
\int_{\Omega} \partial_{n}\left(\frac{h^{\prime \prime}(|\varepsilon(u)|)|\varepsilon(u)|-h^{\prime}(|\varepsilon(u)|)}{|\varepsilon(u)|^{3}}\right)\left(\varepsilon(u): \varepsilon\left(\partial_{k} u\right)\right)\left(\varepsilon(u): \varepsilon\left(\partial_{k n} u\right)\right) \zeta^{3} d x,
$$

which need the smallness of the error of $t h^{\prime \prime}(t)-h^{\prime}(t)$ and the growth control of $h^{\prime \prime \prime}(t)$.

With the help of Proposition 1.1 and 1.2, an argument by Brezis-Gallouet inequality yields that

Theorem 1.1. Suppose that $u \in C^{3}\left(\Omega, \mathbb{R}^{2}\right)$ is a solution of (1.1) and $|\varepsilon(u)| \leq C$, then there exists a constant $r_{1}>0$ such that

$$
\|D u\|_{L^{\infty}\left(T_{2 r} \backslash T_{r}\right)} \leq C r^{-\frac{1}{2}}(\log r)^{\frac{3}{4}}
$$

for all $r>r_{1}$, provided that $\int_{\Omega} h(|D u|) d x<\infty$.

Our paper is organized as follows: in Sec.2 we prove the proof of Theorem 1.1 under the assumptions of Proposition 1.1 and 1.2 by Brezis-Gallouet inequality. In Sect.3 we are aimed to proving Proposition 1.1 by Point-wise Behavior Theorem in [16] and in the last section we complete the proof of Proposition 1.2.

Throughout this paper we adopt the Einstein summation convention, which means that the sum is taken with respect to indices repeated twice. Moreover, throughout 
the remaining section, we denote by $C$ a general positive constant which depends only on known constant coefficients or norms and may be different from line to line.

\section{Proof of Theorem 1.1}

Under the assumptions of Proposition 1.1 and 1.2, we complete the proof of the main theorem.

Proof. Assume that $R_{0}=1$ without loss of generality, and define

$$
\widetilde{u}(\widetilde{x})=r u(r \widetilde{x})=r u(x),
$$

where $x \in T_{2 r} \backslash T_{r}$ and $r$ is large enough. By Lemma 5.4, we have

$$
\|D \widetilde{u}\|_{L^{\infty}\left(T_{2} \backslash T_{1}\right)} \leq C\left(1+\|D \widetilde{u}\|_{H^{1}\left(T_{2} \backslash T_{1}\right)}\right) \sqrt{\log \left(e+\left\|D^{3} \widetilde{u}\right\|_{L^{2}\left(T_{2} \backslash T_{1}\right)}\right)},
$$

And due to scaling, we have

Hence

$$
\begin{aligned}
\|D \widetilde{u}\|_{L^{\infty}\left(T_{2} \backslash T_{1}\right)} & =r^{2}\|D u\|_{L^{\infty}\left(T_{2 r} \backslash T_{r}\right)}, \\
\|D \widetilde{u}\|_{L^{2}\left(T_{2} \backslash T_{1}\right)} & =r\|D u\|_{L^{2}\left(T_{2 r} \backslash T_{r}\right)}, \\
\left\|D^{2} \widetilde{u}\right\|_{L^{2}\left(T_{2} \backslash T_{1}\right)} & =r^{2}\left\|D^{2} u\right\|_{L^{2}\left(T_{2 r} \backslash T_{r}\right)}, \\
\left\|D^{3} \widetilde{u}\right\|_{L^{2}\left(T_{2} \backslash T_{1}\right)} & =r^{3}\left\|D^{3} u\right\|_{L^{2}\left(T_{2 r} \backslash T_{r}\right)} .
\end{aligned}
$$

Due to the Proposition 1.1, there holds

$$
\int_{T_{2 r} \backslash T_{r}}\left|D^{2} u\right|^{2} d x \leq \frac{C \sqrt{\log r}}{r} .
$$

Besides, due to Proposition 1.2, we also get

$$
\int_{T_{2 r} \backslash T_{r}}\left|D^{3} u\right|^{2} d x \leq C .
$$

Then using (2.1), one can get

$$
\begin{aligned}
& r^{2}\|D u\|_{L^{\infty}\left(T_{2 r} \backslash T_{r}\right)} \leq C\left(1+r\|D u\|_{L^{2}\left(T_{2 r} \backslash T_{r}\right)}+r^{2}\left\|D^{2} u\right\|_{L^{2}\left(T_{2 r} \backslash T_{r}\right)}\right) \\
& \times \sqrt{\log \left(e+r^{3}\left\|D^{3} u\right\|_{L^{2}\left(T_{2 r} \backslash T_{r}\right)}\right)} \\
& \leq C\left(1+C r+C r^{\frac{3}{2}}(\log r)^{\frac{1}{4}}\right) \sqrt{\log \left(e+C r^{3}\right)} \\
& \leq C r^{\frac{3}{2}}(\log r)^{\frac{1}{4}} \sqrt{\log r}
\end{aligned}
$$

which implies

$$
\|D u\|_{L^{\infty}\left(T_{2 r} \backslash T_{r}\right)} \leq C r^{-\frac{1}{2}}(\log r)^{\frac{3}{4}} .
$$

The proof of Theorem 1.1 is complete. 


\section{Proof of Proposition 1.1}

Assume that $R_{0}=1$ without loss of generality and $\Omega=\mathbb{R}^{2} \backslash \overline{B_{1}}$. Moreover, $T_{r}=B_{r} \backslash \overline{B_{1}}$ for any $r>1$.

To prove the decay of $D^{2} u$, on one hand we explore the weak maximum principle of the equation (1.1), which is similar to the vorticity form of the Navier-Stokes equations; on the other hand, an obstacle is to deal with the exterior domain and it seems that one can't apply the embedding theorem with scaling domain and the iterative lemma 5.3 simultaneously. Our main idea is to apply the embedding theorem and the iterative lemma 5.3 in different domains. More precisely, we use the embedding theorem in a circular domain with a proportional boundary via weak maximum principle of the equation (1.1), but the iterative lemma is applies in the whole domain.

The proof is divided into two steps, at first we want to prove the $L^{2}$ norm of $D^{2} u$ is bounded and at last we prove the decay of this norm.

First, we introduce the cut-off functions, which will be used in the next proof.

Case I. The choosing of $\phi$. Assume that $\phi(x) \in C_{0}^{\infty}(\Omega)$ with $0 \leq \phi \leq 1$, satisfying that

i) for $r>10, \rho>0$ and $\tau>0$, there holds $\frac{3}{4} r \leq \rho<\tau \leq r$;

ii)

$$
\phi(x)=\phi(|x|)=\left\{\begin{array}{l}
1, \text { in } T_{\rho} \backslash T_{3} ; \\
0, \text { in } T_{2}, \text { and } \Omega \backslash T_{\tau}
\end{array}\right.
$$

iii)

$$
\begin{aligned}
& |D \phi| \leq \frac{C}{\tau-\rho}, \quad\left|D^{2} \phi\right| \leq \frac{C}{(\tau-\rho)^{2}}, \quad \text { as } x \in T_{\tau} \backslash T_{\rho} \\
& |D \phi| \leq C, \quad\left|D^{2} \phi\right| \leq C, \quad \text { as } x \in T_{3} \backslash T_{2}
\end{aligned}
$$

Case 2. The choosing of $\psi$. Assume that $\psi(x) \in C_{0}^{\infty}(\Omega)$ with $0 \leq \psi \leq 1$, satisfying that

i) for $r \gg 10$, there holds

$$
\psi(x)=\psi(|x|)=\left\{\begin{array}{l}
1, \text { in } T_{2 r} \backslash T_{r} ; \\
0, \text { in } T_{\frac{r}{2}}, \text { and } \Omega \backslash T_{3 r}
\end{array}\right.
$$

ii)

$$
|D \psi| \leq \frac{C}{r}, \quad\left|D^{2} \psi\right| \leq \frac{C}{r^{2}} .
$$

Proof. First, we want to obtain Caccioppoli-type inequality by following the same route as in [11] or [25]. Then we estimate the crucial items in more delicate analysis. 
Choose the test function $\varphi_{k}=\partial_{k} u \eta^{2}$, where the cut-off function $\eta \in C_{0}^{\infty}(\Omega)$ with $0 \leq \eta \leq 1$. Multiply (1.1) with $\partial_{k} \varphi_{k}$, and integration by parts yields

$$
\int_{\Omega} \partial_{k} \sigma: \varepsilon\left(\varphi_{k}\right) d x-\int_{\Omega} D \pi \cdot \partial_{k} \varphi_{k} d x-\int_{\Omega} u_{i} \partial_{i} u \cdot \partial_{k} \varphi_{k} d x=0 .
$$

where $\sigma \doteq D H(\varepsilon(u))=\frac{h^{\prime}(|\varepsilon(u)|)}{|\varepsilon(u)|} \varepsilon(u)$.

Using integration by parts again, we obtain

$$
\begin{aligned}
\int_{\Omega} \partial_{k} \sigma: \varepsilon\left(\partial_{k} u\right) \eta^{2} d x= & \int_{\Omega} \sigma: \partial_{k}\left(D \eta^{2} \otimes \partial_{k} u\right) d x+\int_{\Omega} \partial_{k} \pi \operatorname{div}\left(\varphi_{k}\right) d x \\
& +\int_{\Omega} u_{i} \partial_{i} u \cdot \partial_{k} \varphi_{k} \doteq I_{1}+I_{2}+I_{3}
\end{aligned}
$$

where $\otimes$ is the tensor product of vectors.

For $I_{1}$, noticing the relation $\left|D^{2} u(x)\right| \leq 2|D \varepsilon(u)(x)|$, by Young's inequality we have

$$
\begin{aligned}
I_{1} & =\int_{\Omega} \sigma: \partial_{k}\left(D \eta^{2} \otimes \partial_{k} u\right) d x \\
& \leq C\left\{\int_{\Omega} h^{\prime}(|\varepsilon(u)|)|D u|\left(|D \eta|^{2}+\eta\left|D^{2} \eta\right|\right) d x+\int_{\Omega} h^{\prime}(|\varepsilon(u)|)|D \eta|\left|D^{2} u\right| \eta d x\right\} \\
& \leq \delta \int_{\Omega} \frac{h^{\prime}(|\varepsilon(u)|)}{|\varepsilon(u)|}|D \varepsilon(u)|^{2} \eta^{2} d x+C(\delta) \int_{\Omega} h^{\prime}(|\varepsilon(u)|)|\varepsilon(u)||D \eta|^{2} d x \\
& +C \int_{\Omega} h^{\prime}(|\varepsilon(u)|)|D u|\left(|D \eta|^{2}+\eta\left|D^{2} \eta\right|\right) d x \\
& \leq \delta \int_{\Omega} D^{2} H(\varepsilon(u))\left(\varepsilon\left(\partial_{k} u\right),\left(\varepsilon\left(\partial_{k} u\right)\right) \eta^{2} d x+C(\delta) \int_{\Omega} h(|\varepsilon(u)|)|D \eta|^{2} d x\right. \\
& +C \int_{\Omega} h^{\prime}(|\varepsilon(u)|)|D u|\left(|D \eta|^{2}+\eta\left|D^{2} \eta\right|\right) d x .
\end{aligned}
$$

where $\delta>0$, to be decided, and we used the estimates (1.5) and (1.4) in the last step.

For $I_{3}$, we have

$$
\begin{aligned}
I_{3} & =\int_{\Omega} u_{i} \partial_{i} u_{j} \partial_{k}\left(\partial_{k} u_{j} \eta^{2}\right) d x=-\int_{\Omega} \partial_{k}\left(u_{i} \partial_{i} u_{j}\right) \partial_{k} u_{j} \eta^{2} d x \\
& =-\int_{\Omega} \partial_{k} u_{i} \partial_{i} u_{j} \partial_{k} u_{j} \eta^{2} d x-\int_{\Omega} u_{i} \frac{1}{2} \partial_{i}\left(\left|\partial_{k} u_{j}\right|^{2}\right) \eta^{2} d x \\
& =\frac{1}{2} \int_{\Omega}|D u|^{2} u \cdot D \eta^{2} d x
\end{aligned}
$$

where we use $\partial_{k} u_{i} \partial_{i} u_{j} \partial_{k} u_{j}=0$ for divergence free vector $u$ in $2 \mathrm{D}$. 
Next, we use (1.1) to replace $D \pi$, we have

$$
\begin{aligned}
I_{2} & =\int_{\Omega} \partial_{k} \pi d i v\left(\varphi_{k}\right) d x=\int_{\Omega} \partial_{k} \pi \partial_{k} u \cdot D \eta^{2} d x \\
& =-\int_{\Omega} \sigma_{i k} \partial_{i}\left(\partial_{k} u \cdot D \eta^{2}\right) d x-\int_{\Omega} u_{i} \partial_{i} u_{k} \partial_{k} u \cdot D \eta^{2} d x .
\end{aligned}
$$

We estimate (3.4) in the same way as $I_{1}$ and $I_{3}$ :

$$
\begin{aligned}
I_{2} \leq & \delta \int_{\Omega} D^{2} H(\varepsilon(u))\left(\varepsilon\left(\partial_{k} u\right),\left(\varepsilon\left(\partial_{k} u\right)\right) \eta^{2} d x+C(\delta) \int_{\Omega} h(|\varepsilon(u)|)|D \eta|^{2} d x\right. \\
& +C \int_{\Omega} h^{\prime}(|\varepsilon(u)|)|D u|\left(|D \eta|^{2}+\eta\left|D^{2} \eta\right|\right) d x+C \int_{\Omega}|D u|^{2}|u||D \eta| \eta d x .
\end{aligned}
$$

Finally, we observe that

$$
\partial_{k} \sigma: \varepsilon\left(\partial_{k} u\right)=D^{2} H(\varepsilon(u))\left(\varepsilon\left(\partial_{k} u\right), \varepsilon\left(\partial_{k} u\right)\right) .
$$

Recall (3.3) and collect the estimates of $I_{1}, \cdots, I_{3}$, and by choosing $\delta$ small enough we deduce

$$
\begin{aligned}
& \int_{\Omega} D^{2} H(\varepsilon(u))\left(\varepsilon\left(\partial_{k} u\right), \varepsilon\left(\partial_{k} u\right)\right) \eta^{2} d x \leq C \int_{\Omega} h(|\varepsilon(u)|)|D \eta|^{2} d x \\
& +C \int_{\Omega} h^{\prime}(|\varepsilon(u)|)|D u|\left(|D \eta|^{2}+\eta\left|D^{2} \eta\right|\right) d x+C \int_{\Omega}|D u|^{2}|u||D \eta| \eta d x .
\end{aligned}
$$

Note that (1.6), $|\varepsilon(u)| \leq|D u|$ and (1.4) and we get

$$
\begin{aligned}
& \int_{\Omega}\left|\varepsilon\left(\partial_{k} u\right)\right|^{2} \eta^{2} d x \leq C \int_{\Omega} h(|\varepsilon(u)|)|D \eta|^{2} d x \\
& +C \int_{\Omega} h(|D u|)\left(|D \eta|^{2}+\eta\left|D^{2} \eta\right|\right) d x+C \int_{\Omega}|D u|^{2}|u||D \eta| \eta d x .
\end{aligned}
$$

\section{Step I. The bounded estimate.}

In this step, we choose the cut-off function $\eta=\phi$. Note that (3.1) (3.5), and the energy bounded assumption, then we deduce that

$$
\begin{aligned}
\int_{T_{\rho} \backslash T_{2}}\left|\varepsilon\left(\partial_{k} u\right)\right|^{2} d x & \leq C \frac{1}{(\tau-\rho)^{2}}+C \frac{1}{\tau-\rho} \int_{T_{\tau} \backslash T_{\rho} \cup\left(T_{3} \backslash T_{2}\right)}|D u|^{2}|u| \phi d x \\
& \doteq I_{1}^{\prime}+I_{2}^{\prime} .
\end{aligned}
$$

For the term $I_{2}^{\prime}$, noting that $\frac{\tau}{2} \leq \rho$ and $u, D u$ is bounded in $T_{3} \backslash T_{2}$, we have

$$
I_{2}^{\prime} \leq C \frac{1}{\tau-\rho} \int_{T_{\tau} \backslash T_{\frac{\tau}{2}}}|D u|^{2}|u| \phi d x+C \frac{1}{\tau-\rho} \doteq I_{3}^{\prime}+C \frac{1}{\tau-\rho}
$$

Next, we deal with the first term of the right hand. Let

$$
\bar{f}(r)=\frac{1}{2 \pi} \int_{0}^{2 \pi} f(r, \theta) d \theta
$$


then by Wirtinger's inequality (for example, for $p=2$ see Ch II.5 [16]) we have

$$
\int_{0}^{2 \pi}|f-\bar{f}|^{3} d \theta \leq C \int_{0}^{2 \pi}\left|\partial_{\theta} f\right|^{3} d \theta
$$

By Hölder inequality,

$$
I_{3}^{\prime} \leq C \frac{1}{\tau-\rho}\left(\int_{T_{\tau} \backslash T_{\frac{\tau}{2}}}|D u|^{3} \phi d x\right)^{\frac{2}{3}}\left(\int_{T_{\tau} \backslash T_{\frac{\tau}{2}}}|u-\bar{u}+\bar{u}|^{3} \phi d x\right)^{\frac{1}{3}}
$$

Using (3.6) and Lemma 5.1 we derive

$$
\begin{aligned}
\int_{T_{\tau} \backslash T_{\frac{\tau}{2}}}|u|^{3} d x \leq & \left(\int_{\frac{\tau}{2}<r^{\prime}<\tau} \int_{0}^{2 \pi}\left|u\left(r^{\prime}, \theta\right)-\bar{u}\right|^{3} d \theta r^{\prime} d r^{\prime}\right) \\
& +\int_{T_{\tau} \backslash T_{\frac{\tau}{2}}}\left(\int_{0}^{2 \pi} u(r, \theta) d \theta\right)^{3} d x \\
\leq & C r^{3}\left(\int_{\frac{\tau}{2}<r^{\prime}<\tau} \frac{1}{r^{\prime 3}} \int_{0}^{2 \pi}\left|\partial_{\theta} u\right|^{3} d \theta r^{\prime} d r^{\prime}\right)+C(\ln r)^{\frac{3}{2}} r^{2} \\
\leq & C r^{3} \int_{T_{\tau} \backslash T_{\frac{\tau}{2}}}|D u|^{3} d x+C(\ln r)^{\frac{3}{2}} r^{2}
\end{aligned}
$$

for $r>2 r_{0}\left(r_{0}\right.$ is a constant in Lemma 5.1), since (1.3).

Hence, by using $(\ln r)^{\frac{3}{2}} \leq C r^{\frac{1}{2}}$ there holds

$$
I_{3}^{\prime} \leq C \frac{r}{\tau-\rho} \int_{T_{\tau} \backslash T_{\frac{\tau}{2}}}|D u|^{3} d x+C r^{-\frac{1}{2}}
$$

Recall that the following Poincaré-Sobolev inequality holds(see, for example, Theorem 8.11 and 8.12 [22])

$$
\|w\|_{L^{3}\left(B_{\tau} \backslash B_{\tau / 2}\right)} \leq C\|D w\|_{L^{2}\left(B_{\tau} \backslash B_{\tau / 2}\right)}^{\frac{1}{3}}\|w\|_{L^{2}\left(B_{\tau}\right)}^{\frac{2}{3}}+C \tau^{-\frac{1}{3}}\|w\|_{L^{2}\left(B_{\tau} \backslash B_{\tau / 2}\right)},
$$

which implies that

$$
\begin{aligned}
I_{3}^{\prime} & \leq C \frac{r}{\tau-\rho}\left(\int_{T_{\tau} \backslash T_{\frac{\tau}{2}}}\left|D^{2} u\right|^{2} d x\right)^{\frac{1}{2}}\left(\int_{T_{\tau} \backslash T_{\frac{\tau}{2}}}|D u|^{2} d x\right)+C \frac{1}{\tau-\rho}\left(\int_{T_{\tau} \backslash T_{\frac{\tau}{2}}}|D u|^{2} d x\right)^{\frac{3}{2}} \\
& \leq \frac{1}{16}\left(\int_{T_{\tau} \backslash T_{\frac{\tau}{2}}}\left|D^{2} u\right|^{2} d x\right)+C \frac{r^{2}}{(\tau-\rho)^{2}}
\end{aligned}
$$

Collecting the estimates of $I_{1}^{\prime}, \cdots, I_{3}^{\prime}$, we get

$$
\int_{T_{\rho} \backslash T_{2}}\left|\varepsilon\left(\partial_{k} u\right)\right|^{2} d x \leq \frac{1}{16}\left(\int_{T_{\tau} \backslash T_{\frac{\tau}{2}}}\left|D^{2} u\right|^{2} d x\right)+C \frac{1}{(\tau-\rho)}+C \frac{r^{2}}{(\tau-\rho)^{2}}
$$


Then by applying $\left|D^{2} u(x)\right| \leq 2|D \varepsilon(u)(x)|$ again, Lemma 5.3 yields

$$
\int_{T_{\frac{3}{4} r} \backslash T_{2}}\left|D^{2} u\right|^{2} d x \leq C
$$

Finally, by taking $r \rightarrow \infty$, we arrive at

$$
\int_{\Omega \backslash T_{2}}\left|D^{2} u\right|^{2} d x \leq C
$$

\section{Step II. The decay estimate.}

In this step, we choose the cut-off function $\eta=\psi$. Note that (3.2) (3.5), and the energy bounded assumption, then we deduce that

$$
\begin{aligned}
\int_{T_{2 r} \backslash T_{r}}\left|\varepsilon\left(\partial_{k} u\right)\right|^{2} d x & \leq C \frac{1}{r^{2}}+C \frac{1}{r} \int_{T_{3 r} \backslash T_{r / 2}}|D u|^{2}|u| \phi d x \\
& \doteq C \frac{1}{r^{2}}+I_{4}^{\prime} .
\end{aligned}
$$

Due to $D u \in L^{2}(\Omega)$ and (3.9), it follows from Gagliardo-Nirenberg inequality that

$$
\|D u\|_{L^{p}\left(\Omega \backslash T_{3}\right)}<\infty, \quad \forall p>2 .
$$

Thus with the help of Lemma 5.2, we have

$$
|u(x)| \leq \sqrt{\ln (|x|)}
$$

for a sufficient large constant, still denoted by $r_{0}$, and $|x| \geq r_{0}$. Consequently,

$$
\int_{T_{2 r} \backslash T_{r}}\left|\varepsilon\left(\partial_{k} u\right)\right|^{2} d x \leq C \frac{\sqrt{\ln r}}{r},
$$

which implies the required inequality (1.7).

\section{Proof of Proposition 1.2}

In this section, we introduce another cut-off functions, which will be used in the next proof.

Case III. The choosing of $\zeta$. First, we introduce a cut-off function $\zeta \in C_{0}^{\infty}(\Omega)$ with $0 \leq \zeta \leq 1$, satisfying that

i) for $r>2 r_{0}, \rho>0$ and $\tau>0$, there holds $\frac{3}{4} r \leq \rho<\tau \leq r$;

ii)

$$
\zeta(x)=\zeta(|x|)=\left\{\begin{array}{l}
1, \text { in } T_{2 \rho} \backslash T_{r+\frac{r-\rho}{2}} ; \\
0, \text { in } T_{r+\frac{r-\tau}{2}}, \text { and } \Omega \backslash T_{2 \tau}
\end{array}\right.
$$

iii) $|D \zeta| \leq \frac{C}{\tau-\rho},\left|D^{2} \zeta\right| \leq \frac{C}{(\tau-\rho)^{2}}$. 
Proof. For any $\zeta \in C_{0}^{\infty}(\Omega)$ with $0 \leq \zeta \leq 1$, letting $\varphi_{k}=\partial_{k} \Delta u \zeta^{3}$ with $k=1,2$, we multiply (1.1) with $\partial_{k} \varphi_{k}$ and use integration by parts to obtain

$$
\int_{\Omega} \partial_{k} \sigma: \varepsilon\left(\varphi_{k}\right) d x-\int_{\Omega} D \pi \cdot \partial_{k} \varphi_{k} d x-\int_{\Omega} u_{i} \partial_{i} u \cdot \partial_{k} \varphi_{k} d x=0 .
$$

where $\sigma:=D H(\varepsilon(u)):=\frac{h^{\prime}(|\varepsilon(u)|)}{|\varepsilon(u)|} \varepsilon(u)$.

Using integration by parts again, by $\varphi_{k}=\partial_{k} \Delta u \zeta^{3}$ we get

$$
\begin{aligned}
\int_{\Omega} \partial_{k} \sigma: \varepsilon\left(\partial_{k} \Delta u\right) \zeta^{3} d x= & \int_{\Omega} \sigma: \partial_{k}\left(D \zeta^{3} \otimes \partial_{k} \Delta u\right) d x \\
& +\int_{\Omega} \partial_{k} \pi \operatorname{div}\left(\varphi_{k}\right) d x+\int_{\Omega} u_{i} \partial_{i} u \cdot \partial_{k} \varphi_{k} d x .
\end{aligned}
$$

Next we deal with every term of (4.2):

$$
\begin{aligned}
& \int_{\Omega} \partial_{k} \sigma: \varepsilon\left(\partial_{k} \Delta u\right) \zeta^{3} d x \\
& =\int_{\Omega} \frac{h^{\prime \prime}(|\varepsilon(u)|)|\varepsilon(u)|-h^{\prime}(|\varepsilon(u)|)}{|\varepsilon(u)|^{2}} \frac{\varepsilon(u): \varepsilon\left(\partial_{k} u\right)}{|\varepsilon(u)|} \varepsilon(u): \varepsilon\left(\partial_{k} \Delta u\right) \zeta^{3} d x \\
& +\int_{\Omega} \frac{h^{\prime}(|\varepsilon(u)|)}{|\varepsilon(u)|} \varepsilon\left(\partial_{k} u\right): \varepsilon\left(\partial_{k} \Delta u\right) \zeta^{3} d x \\
& =-\int_{\Omega} \frac{h^{\prime \prime}(|\varepsilon(u)|)|\varepsilon(u)|-h^{\prime}(|\varepsilon(u)|)}{|\varepsilon(u)|^{2}} \frac{\varepsilon(u): \varepsilon\left(\partial_{k n} u\right)}{|\varepsilon(u)|} \varepsilon(u): \varepsilon\left(\partial_{k n} u\right) \zeta^{3} d x \\
& -\int_{\Omega} \frac{h^{\prime}(|\varepsilon(u)|)}{|\varepsilon(u)|} \varepsilon\left(\partial_{k n} u\right): \varepsilon\left(\partial_{k n} u\right) \zeta^{3} d x \\
& -\int_{\Omega} \partial_{n}\left(\frac{h^{\prime \prime}(|\varepsilon(u)|)|\varepsilon(u)|-h^{\prime}(|\varepsilon(u)|)}{|\varepsilon(u)|^{3}}\right)\left(\varepsilon(u): \varepsilon\left(\partial_{k} u\right)\right)\left(\varepsilon(u): \varepsilon\left(\partial_{k n} u\right)\right) \zeta^{3} d x \\
& -\int_{\Omega} \frac{h^{\prime \prime}(|\varepsilon(u)|)|\varepsilon(u)|-h^{\prime}(|\varepsilon(u)|)}{|\varepsilon(u)|^{2}} \frac{\varepsilon\left(\partial_{n} u\right): \varepsilon\left(\partial_{k} u\right)}{|\varepsilon(u)|} \varepsilon(u): \varepsilon\left(\partial_{k n} u\right) \zeta^{3} d x \\
& -\int_{\Omega} \frac{h^{\prime \prime}(|\varepsilon(u)|)|\varepsilon(u)|-h^{\prime}(|\varepsilon(u)|)}{|\varepsilon(u)|^{2}} \frac{\varepsilon(u): \varepsilon\left(\partial_{k} u\right)}{|\varepsilon(u)|} \varepsilon\left(\partial_{n} u\right): \varepsilon\left(\partial_{k n} u\right) \zeta^{3} d x \\
& -\int_{\Omega} \partial_{n}\left(\frac{h^{\prime}(|\varepsilon(u)|)}{|\varepsilon(u)|}\right) \varepsilon\left(\partial_{k} u\right): \varepsilon\left(\partial_{k n} u\right) \zeta^{3} d x \\
& -\int_{\Omega} \frac{h^{\prime \prime}(|\varepsilon(u)|)|\varepsilon(u)|-h^{\prime}(|\varepsilon(u)|)}{|\varepsilon(u)|^{2}} \frac{\varepsilon(u): \varepsilon\left(\partial_{k} u\right)}{|\varepsilon(u)|} \varepsilon(u): \varepsilon\left(\partial_{k n} u\right) \partial_{n}\left(\zeta^{3}\right) d x \\
& -\int_{\Omega} \frac{h^{\prime}(|\varepsilon(u)|)}{|\varepsilon(u)|} \varepsilon\left(\partial_{k} u\right): \varepsilon\left(\partial_{k n} u\right) \partial_{n}\left(\zeta^{3}\right) d x \\
& \doteq-J_{1}-\cdots-J_{8}
\end{aligned}
$$


Note that (1.5), and we have $J_{1} \geq 0$. By (1.9), we have

$$
J_{2} \geq \int_{\Omega}\left|\varepsilon\left(\partial_{k n} u\right)\right|^{2} \zeta^{3} d x
$$

Using $\left|D^{3} u(x)\right| \leq 2\left|\varepsilon\left(D^{2} u\right)(x)\right|$, we have

$$
J_{2} \geq \frac{1}{4} \int_{\Omega}\left|D^{3} u\right|^{2} \zeta^{3} d x
$$

Then

$$
\begin{aligned}
& \frac{1}{4} \int_{\Omega}\left|D^{3} u\right|^{2} \zeta^{3} d x \leq \int_{\Omega} \partial_{k} \sigma:\left(D \zeta^{3} \otimes \partial_{k} \Delta u\right) d x \\
& -\int_{\Omega} \partial_{k} \pi \operatorname{div}\left(\varphi_{k}\right) d x-\int_{\Omega} u_{i} \partial_{i} u \cdot \partial_{k} \varphi_{k} d x-J_{3}-\cdots-J_{8} .
\end{aligned}
$$

For $J_{3}$, using (1.11), Young's inequality and $|\varepsilon(u)| \leq C$, we have

$$
\begin{aligned}
J_{3} & =\int_{\Omega} \partial_{n}\left(\frac{h^{\prime \prime}(|\varepsilon(u)|)|\varepsilon(u)|-h^{\prime}(|\varepsilon(u)|)}{|\varepsilon(u)|^{3}}\right)\left(\varepsilon(u): \varepsilon\left(\partial_{k} u\right)\right)\left(\varepsilon(u): \varepsilon\left(\partial_{k n} u\right)\right) \zeta^{3} d x \\
& \leq C \int_{\Omega}\left(|\varepsilon(u)|^{\gamma_{0}}+1+|\varepsilon(u)|^{\gamma_{1}-1}+|\varepsilon(u)|^{\gamma_{2}-1}\right)\left|D^{2} u\right|^{2}\left|D^{3} u\right| \zeta^{3} d x \\
& \leq C \int_{\Omega}\left|D^{2} u\right|^{4} \zeta^{4} d x+\frac{1}{64} \int_{\Omega}\left|D^{3} u\right|^{2} \zeta^{2} d x .
\end{aligned}
$$

where we used

$$
\left|\frac{h^{\prime \prime}(|\varepsilon(u)|)|\varepsilon(u)|-h^{\prime}(|\varepsilon(u)|)}{|\varepsilon(u)|^{2}}\right| \leq C\left(|\varepsilon(u)|^{\gamma_{1}-1}+|\varepsilon(u)|^{\gamma_{2}-1}\right)
$$

since (1.10).

Similarly, $J_{6}$ can be estimated immediately as

$$
\begin{aligned}
J_{6} & =\int_{\Omega} \frac{h^{\prime \prime}(|\varepsilon(u)|)|\varepsilon(u)|-h^{\prime}(|\varepsilon(u)|)}{|\varepsilon(u)|^{2}} \partial_{n}|\varepsilon(u)| \varepsilon\left(\partial_{k} u\right): \varepsilon\left(\partial_{k n} u\right) \zeta^{3} d x \\
& \leq C \int_{\Omega}\left|D^{2} u\right|^{4} \zeta^{4} d x+\frac{1}{64} \int_{\Omega}\left|D^{3} u\right|^{2} \zeta^{2} d x .
\end{aligned}
$$

For $J_{4}$ and $J_{5}$, in the same way we have

$$
\begin{aligned}
J_{4}+J_{5} & \leq C \int_{\Omega}\left(|\varepsilon(u)|^{\gamma_{2}-1}+|\varepsilon(u)|^{\gamma_{1}-1}\right)\left|D^{2} u\right|^{2}\left|D^{3} u\right| \zeta^{3} d x \\
& \leq C \int_{\Omega}\left|D^{2} u\right|^{4} \zeta^{4} d x+\frac{1}{64} \int_{\Omega}\left|D^{3} u\right|^{2} \zeta^{2} d x .
\end{aligned}
$$

For $J_{7}$ and $J_{8}$, using (4.4) and Young inequality again, we get

$$
J_{7}+J_{8} \leq C \int_{\Omega}\left(1+|\varepsilon(u)|^{\gamma_{2}}+|\varepsilon(u)|^{\gamma_{1}}\right)\left|D^{2} u\right|\left|D^{3} u\right| \zeta^{2}|D \zeta| d x
$$




$$
\leq C \int_{\Omega}\left|D^{2} u\right|^{2} \zeta^{2}|D \zeta|^{2} d x+\frac{1}{64} \int_{\Omega}\left|D^{3} u\right|^{2} \zeta^{2} d x
$$

Next, we estimate the first three terms of the right hand in (4.3). Firstly, using (1.9) and (1.10) we get

$$
\begin{aligned}
A_{1} & \doteq \int_{\Omega} \partial_{k} \sigma:\left(D \zeta^{3} \otimes \partial_{k} \Delta u\right) d x \\
& =\int_{\Omega} \partial_{k}\left(\frac{h^{\prime}(|\varepsilon(u)|)}{|\varepsilon(u)|} \varepsilon(u)\right):\left(D \zeta^{3} \otimes \partial_{k} \Delta u\right) d x \\
& \leq C \int_{\Omega}\left(1+|\varepsilon(u)|^{\gamma_{2}}\right)\left|D^{2} u\right| \zeta^{2}|D \zeta|\left|D^{3} u\right| d x \\
& \leq C \int_{\Omega}\left|D^{2} u\right|^{2} \zeta^{2}|D \zeta|^{2} d x+\frac{1}{64} \int_{\Omega}\left|D^{3} u\right|^{2} \zeta^{2} d x
\end{aligned}
$$

Besides,

$$
\begin{aligned}
A_{2} & \doteq \int_{\Omega} u_{i} \partial_{i} u \cdot \partial_{k} \varphi_{k} d x \\
& =-\int_{\Omega} \partial_{k} u_{i} \partial_{i} u_{j} \partial_{k} \Delta u_{j} \zeta^{3} d x-\int_{\Omega} u_{i} \partial_{k i} u_{j} \partial_{k} \Delta u_{j} \zeta^{3} d x \\
& \leq C \int_{\Omega}|D u|^{2}\left|D^{3} u\right| \zeta^{3} d x+C \int_{\Omega}|u|\left|D^{2} u\right|\left|D^{3} u\right| \zeta^{3} d x \\
& \leq \frac{1}{64} \int_{\Omega}\left|D^{3} u\right|^{2} \zeta^{2} d x+C \int_{\Omega}|u|^{2}\left|D^{2} u\right|^{2} \zeta^{4} d x+C \int_{\Omega}|D u|^{4} \zeta^{4} d x
\end{aligned}
$$

Finally, we estimate $A_{3}=\int_{\Omega} \partial_{k} \pi \operatorname{div}\left(\varphi_{k}\right) d x$. According the equation of (1.1),

$$
\begin{aligned}
\int_{\Omega} \partial_{k} \pi \operatorname{div}\left(\varphi_{k}\right) d x & =\int_{\Omega} \partial_{k} \pi \partial_{k} \Delta u \cdot D \zeta^{3} d x \\
& =\int_{\Omega} \partial_{i} \sigma_{i k} \partial_{k} \Delta u \cdot D \zeta^{3} d x-\int_{\Omega} u_{i} \partial_{i} u_{k} \partial_{k} \Delta u \cdot D \zeta^{3} d x
\end{aligned}
$$

whose estimates are similar to $A_{1}$ and $A_{2}$, i.e.

$$
\begin{aligned}
A_{3} \leq & \frac{1}{64} \int_{\Omega}\left|D^{3} u\right|^{2} \zeta^{2} d x+C \int_{\Omega}\left|D^{2} u\right|^{2} \zeta^{2}|D \zeta|^{2} d x \\
& +C \int_{\Omega}|u|^{2}|D u|^{2}|D \zeta|^{2} \zeta^{2} d x
\end{aligned}
$$

Recalling (4.3), and combining $J_{3}, \cdots, J_{8}$, (4.5), (4.6) and (4.7), we have

$$
\begin{aligned}
\frac{1}{4} \int_{\Omega}\left|D^{3} u\right|^{2} \zeta^{3} d x & \leq \frac{1}{8} \int_{\Omega}\left|D^{3} u\right|^{2} \zeta^{2} d x+C \int_{\Omega}|u|^{2}\left|D^{2} u\right|^{2} \zeta^{4} d x \\
& +C \int_{\Omega}|D u|^{4} \zeta^{4} d x+C \int_{\Omega}\left|D^{2} u\right|^{2} \zeta^{2}|D \zeta|^{2} d x
\end{aligned}
$$




$$
+C \int_{\Omega}\left|D^{2} u\right|^{4} \zeta^{4} d x+C \int_{\Omega}|u|^{2}|D u|^{2}|D \zeta|^{2} \zeta^{2} d x .
$$

Note that the bounded energy integral and (3.10), i.e. $D u \in L^{p}(\Omega)$ for any $p \geq 2$, and we derive

$$
\begin{aligned}
\frac{1}{4} \int_{\Omega}\left|D^{3} u\right|^{2} \zeta^{3} d x & \leq \frac{1}{8} \int_{\Omega}\left|D^{3} u\right|^{2} \zeta^{2} d x+C \frac{(\ln r)^{\frac{3}{2}}}{r} \\
& +C+C \frac{\sqrt{\ln r}}{r(\tau-\rho)^{2}}+C \frac{\ln r}{(\tau-\rho)^{2}} \\
& +C \int_{\Omega}\left|D^{2} u\right|^{4} \zeta^{4} d x
\end{aligned}
$$

where we also used the decay estimate in Proposition 1.1. Next we deal with the last term in the right hand. Due to Gagliardo-Nirenberg inequality,

$$
C \int_{\Omega}\left|D^{2} u \zeta\right|^{4} d x \leq C_{0}\left\|D^{2} u \zeta\right\|_{L^{2}(\Omega)}^{2}\left\|D\left(D^{2} u \zeta\right)\right\|_{L^{2}(\Omega)}^{2},
$$

where $C_{0}$ is an absolute constant. Using Proposition 1.1 again, we have

$$
\begin{aligned}
\int_{\Omega}\left|D^{2} u \zeta\right|^{4} d x & \leq C_{0} C \frac{\sqrt{\ln r}}{r} \int_{\Omega}\left|D^{3} u\right|^{2} \zeta^{2} d x+C \frac{\ln r}{r^{2}(\tau-\rho)^{2}} \\
& \leq \frac{1}{16} \int_{\Omega}\left|D^{3} u\right|^{2} \zeta^{2} d x+C \frac{\ln r}{r^{2}(\tau-\rho)^{2}}
\end{aligned}
$$

provided that $r_{0}$ is large enough.

Combining (4.8) and (4.9), by (4.1) we deduce

$$
\begin{aligned}
\int_{T_{2 \rho} \backslash T_{r+\frac{r-\rho}{2}}}\left|D^{3} u\right|^{2} d x & \leq \frac{3}{4} \int_{T_{2 \tau} \backslash T_{r+\frac{r-\tau}{2}}}\left|D^{3} u\right|^{2} d x+C \frac{(\ln r)^{\frac{3}{2}}}{r} \\
& +C+C \frac{\sqrt{\ln r}}{r(\tau-\rho)^{2}}+C \frac{\ln r}{(\tau-\rho)^{2}}+C \frac{\ln r}{r^{2}(\tau-\rho)^{2}}
\end{aligned}
$$

Applying Lemma 5.3, we have

$$
\begin{aligned}
\int_{T_{2 \rho} \backslash T_{r+\frac{r-\rho}{2}}}\left|D^{3} u\right|^{2} d x \leq & C \frac{(\ln r)^{\frac{3}{2}}}{r} \\
& +C+C \frac{\sqrt{\ln r}}{r(\tau-\rho)^{2}}+C \frac{\ln r}{(\tau-\rho)^{2}}+C \frac{\ln r}{r^{2}(\tau-\rho)^{2}},
\end{aligned}
$$

by taking $\rho=\frac{3}{4} r$ and $\tau=r$, which implies that

$$
\int_{T_{\frac{3}{2} r} \backslash T_{\frac{9}{8} r}}\left|D^{3} u\right|^{2} d x \leq C \frac{(\ln r)^{\frac{3}{2}}}{r}+C \frac{\sqrt{\ln r}}{r^{3}}+\frac{\ln r}{r^{2}}+C
$$




$$
\leq C
$$

which implies the required inequality (1.2).

\section{ApPENDiX}

In the proof of Theorem, we need the following known lemmas.

First, let us recall a result of Gilbarg-Weinberger in [18] about the decay of functions with finite Dirichlet integrals.

Lemma 5.1 (Lemma 2.1, 2.2, [18]). Let a $C^{1}$ vector-valued function $f(x)=\left(f_{1}, f_{2}\right)(x)=$ $f(r, \theta)$ with $r=|x|$ and $x_{1}=r \cos \theta$. There holds finite Dirichlet integral in the range $r>r_{0}$, that is

$$
\int_{r>r_{0}}|D f|^{2} d x d y<\infty
$$

Then, we have

$$
\lim _{r \rightarrow \infty} \frac{1}{\ln r} \int_{0}^{2 \pi}|f(r, \theta)|^{2} d \theta=0 .
$$

If, furthermore, we assume $D f \in L^{p}\left(\mathbb{R}^{2}\right)$ for some $2<p<\infty$, then the above decay property can be improved to be point-wise uniformly. More precisely, we have

Lemma 5.2 (Point-wise Behavior Theorem, Theorem II.9.1 [16]). Let $\Omega \subset \mathbb{R}^{2}$ be an exterior domain and let

$$
D f \in L^{2} \cap L^{p}(\Omega)
$$

for some $2<p<\infty$. Then

$$
\lim _{|x| \rightarrow \infty} \frac{|f(x)|}{\sqrt{\ln (|x|)}}=0,
$$

uniformly.

We also need a Giaquinta's iteration lemma [17, Lemma 3.1].

Lemma 5.3 (Lemma 3.1 [17]). Let $f(r)$ be a non-negative bounded function on $\left[R_{0}, R_{1}\right] \subset \mathbb{R}_{+}$. If there are negative constants $A, B, D$ and positive exponents $b<a$ and a parameter $\theta \in(0,1)$ such that for all $R_{0} \leq \rho<\tau \leq R_{1}$

$$
f(\rho) \leq \theta f(\tau)+\frac{A}{(\tau-\rho)^{a}}+\frac{B}{(\tau-\rho)^{b}}+D,
$$

then for all $R_{0} \leq \rho<\tau \leq R_{1}$

$$
f(\rho) \leq C(a, \theta)\left[\frac{A}{(\tau-\rho)^{a}}+\frac{B}{(\tau-\rho)^{b}}+D\right] .
$$


At last, we introduce the Brezis-Gallouet inequality (see Lemma 2 in [7], or Lemma 3.1 in [8]).

Lemma 5.4. Let $f \in H^{2}(\Omega)$ where $\Omega$ is a bounded domain or an exterior domain with compact smooth boundary. Then there exists a constant $C_{\Omega}$ depending only on $\Omega$, such that

or

$$
\|f\|_{L^{\infty}(\Omega)} \leq C_{\Omega}\|f\|_{H^{1}(\Omega)} \ln ^{\frac{1}{2}}\left(e+\frac{\left\|D^{2} f\right\|_{L^{2}(\Omega)}}{\|f\|_{H^{1}(\Omega)}}\right),
$$

$$
\|f\|_{L^{\infty}(\Omega)} \leq C_{\Omega}\left(1+\|f\|_{H^{1}(\Omega)}\right) \ln ^{\frac{1}{2}}\left(e+\left\|D^{2} f\right\|_{L^{2}(\Omega)}\right) .
$$

Note that the second inequality can be obtained immediately from the first one by arguments whether $\|f\|_{H^{1}(\Omega)}<1$, and we omitted it.

\section{ACKNOWLEDGMENTS}

W. Wang was supported by NSFC under grant 11671067 and "the Fundamental Research Funds for the Central Universities".

\section{REFERENCES}

[1] C. J. Amick, On Leray's problem of steady Navier-Stokes flow past a body, Acta Math. 161 (1988), 71-130.

[2] Apushkinskaya, Darya; Bildhauer, Michael; Fuchs, Martin Steady states of anisotropic generalized Newtonian fluids. J. Math. Fluid Mech. 7 (2005), no. 2, 261-297.

[3] Beirão da Veiga, H.: On the regularity of flows with Ladyzhenskaya shear dependent viscosity and slip and non-slip boundary conditions. Commun. Pure Appl. Math. 58, 552-77 (2005)

[4] Bildhauer, M.; Fuchs, M. Twodimensional anisotropic variational problems. Calc. Var. Partial Differential Equations 16 (2003), no. 2, 177-186.

[5] Bildhauer,M., Fuchs,M., Zhang,G.: Liouville-Type theorem for steady flows of degenerate power law fluids in the plane. J. Math. Fluid Mech. 15, 2013, 583-616.

[6] Bildhauer, M., Fuchs, M., Zhong, X., A lemma on the higher integrability of functions with applications to the regularity theory of two dimensional generalized Newtonian fluids. Manuscr. Math. 116(2), 135-156 (2005)

[7] H. Brezis and T. Gallouet, Nonlinear Schrodinger evolution equations, Nonlinear Anal. 4 (1980), no. 4, 677-681.

[8] B. Carrillo, X. Pan, Q. Zhang, Decay and vanishing of some axially symmetric D-solutions of the Navier-Stokes equations, arXiv:1801.07420 [math.AP].

[9] Frehse, J., Málek, J., Steinhauer, M.: On analysis of steady flows of fluids with shear dependent viscosity based on the Lipschitz truncation method. SIAM J. Math. Anal. 34(5), 1064-1083 (2003) 
[10] M. Fuchs, Stationary flows of shear thickening fluids in 2D. J. Math. Fluid Mech. 14 (2012), no. 1, 43-54.

[11] M. Fuchs, Liouville theorems for stationary flows of shear thickening fluids in the plane. J. Math. Fluid Mech. 14 (2012), no. 3, 421-444.

[12] Fuchs, M., and G. Seregin, Variational methods for problems from plasticity theory and for generalized Newtonian fluids. - Lecture Notes in Math. 1749, Springer-Verlag, Berlin, 2000.

[13] M. Fuchs, X. Zhong, A note on a Liouville type result of Gilbarg and Weinberger for the stationary Navier-Stokes equations in 2D. Problems in mathematical analysis. No. 60. J. Math. Sci. (N.Y.) 178 (2011), no. 6, 695-703.

[14] Galdi, G.: An introduction to the mathematical theory of the Navier-Stokes equations vol. I. Springer Tracts in Natural Philosophy vol. 38. Springer, Berlin (1994)

[15] Galdi, G.: An introduction to the mathematical theory of the Navier-Stokes equations vol. II. Springer Tracts in Natural Philosophy, vol. 39. Springer, Berlin (1994)

[16] G. P. Galdi, An introduction to the mathematical theory of the Navier- Stokes equations. Steady-state problems. Second edition. Springer Monographs in Mathematics. Springer, New York, 2011. xiv+1018 pp.

[17] M. Giaquinta, Multiple Integrals in the Calculus of Variations and Nonlinear Elliptic Systems, Princeton University Press, Princeton, New Jersey, (1983).

[18] Gilbarg, D.; Weinberger, H. F., Asymptotic properties of steady plane solutions of the Navier-Stokes equations with bounded Dirichlet integral. Ann. Scuola Norm. Sup. Pisa Cl. Sci. (4) 5 (1978), no. 2, 381-404.

[19] Jin, Bum Ja; Kang, Kyungkeun, Liouville theorem for the steady-state nonNewtonian Navier-Stokes equations in two dimensions. J. Math. Fluid Mech. 16 (2014), no. 2, 275-292.

[20] Ladyzhenskaya, O.A.: The mathematical theory of viscous incompressible flow. Gordon And Breach, New York (1969)

[21] J. Leray, Étude de diverses équations intégrales non linéaire et de quelques problèmes que pose l'hydrodynamique, J. Math. Pures Appl., 12 (1933), 1-82.

[22] E. H. Lieb, M. Loss, Analysis, second edition, Amer. Math. Soc., Providence, RI (2001).

[23] K. Pileckas, R. Russo, On the existence of vanishing at infinity symmetric solutions to the plane stationary exterior Navier-Stokes problem, Math. Ann. 352 (2012), no. 3, 643-658.

[24] A. Russo, A note on the exterior two-dimensional steady-state Navier-Stokes problem. J. Math. Fluid Mech. 11 (2009), no. 3, 407-414.

[25] G. Zhang, A note on Liouville theorem for stationary flows of shear thickening fluids in the plane. J. Math. Fluid Mech. 15 (2013), no. 4, 771-782. 
[26] G. Zhang, Liouville theorems for stationary flows of shear thickening fluids in 2D. Ann. Acad. Sci. Fenn. Math. 40 (2015), no. 2, 889-905.

(Shuai Li) School of Mathematical Sciences, Dalian University of Technology, DALIAN, 116024, ChINA

E-mail address: Is21701048@163.com

(Tao Wang) School of Mathematical Sciences, Dalian University of Technology, DALIAN, 116024, CHINA

E-mail address: muyuwt@163.com

(Wendong Wang) School of Mathematical Sciences, Dalian University of TechnolOGy, Dalian, 116024, China

E-mail address: wendong@dlut.edu.cn 